\title{
Forwarding Strategies for Applications in Named Data Networking
}

\author{
Hila Ben Abraham and Patrick Crowley \\ Computer Science and Engineering \\ Washington University in St. Louis \\ \{hila, pcrowley\}@wustl.edu
}

\begin{abstract}
Named Data Networking (NDN), an information-centric Internet architecture, introduces a new forwarding model, in which the forwarding plane can choose between multiple interfaces when forwarding a packet. While the forwarding module brings new opportunities it also introduces challenges when the application's performance or correctness is affected by a conflict between the application design and the assigned forwarding strategy. In this paper we demonstrate the impact of the forwarding strategy decision on the performance and correctness of NDN applications.
\end{abstract}

\section{INTRODUCTION}

Named Data Networking (NDN) [1] is a consumer-driven architecture that focuses on retrieving a content according to its name. To request a content item, a consumer expresses an Interest packet, which is forwarded in the network until it can be satisfied by a router's Content Store (CS), or by an application that serves as the content producer. The content is returned to the consumer in a Data packet that follows the reverse path of the Interest packet.

As in IP, the Forwarding Information Base (FIB) table is used to determine the packet's next hop. An entry in the NDN FIB consists of a namespace and one or more possible faces. Each face represents an interface to a possible next hop. When the faces list consists of multiple faces, the forwarding plane needs to decide on which face(s) to forward the interest. The forwarding decision is determined by the selected forwarding strategy of the requested namespace.

Two Information-centric network (ICN) prototypes, the NDN forwarder (NFD) [2] and the CCNx project [3] provide an API for NDN or CCN applications. These prototypes allow pairing a forwarding strategy with an application namespace, and therefore affect the way the application packets are forwarded in the network. A developer can choose an existing forwarding strategy, or, alternatively, develop a new one to satisfy application-specific needs. While this brings new opportunities and advantages, it can pose challenges.

Permission to make digital or hard copies of part or all of this work for personal or classroom use is granted without fee provided that copies are not made or distributed for profit or commercial advantage and that copies bear this notice and the full citation on the first page. Copyrights for third-party components of this work must be honored. For all other uses, contact the owner/author(s).

ANCS '16 March 17-18, 2016, Santa Clara, CA, USA

(C) 2016 Copyright held by the owner/author(s).

ACM ISBN 978-1-4503-4183-7/16/03.

DOI: http://dx.doi.org/10.1145/2881025.2889475

\section{FORWARDING AND APPLICATIONS}

Existing forwarding strategies have different design approaches. First, if the FIB entry consists of multiple faces, the forwarding strategy must decide on which faces an Interest should be sent. The strategy may choose the best performing face, a subset of possible faces or all available faces. The decision can be made according to the face cost, which is determined by the routing protocol, or according to the face rank, which is determined by previous measurements of the forwarding strategy. Second, the forwarding strategy must decide how to react when a Data packet is not received on time: 1) Drop the Interest packet. 2) Retransmit the packet on the same or a different face(s). 3) Reply with a special NACK packet to the previous hop. The application developer must be aware of the approach taken by the forwarding strategy to better decide whether an application retransmission is required. Third, in NDN, Interest and data packets are forwarded on the same path (but in reverse direction). Therefore, the forwarding strategy knows which downstream faces work, and how well they work. An Adaptive Forwarding strategy records the performance of the faces to improve future decisions. A Static Forwarding strategy relies exclusively on the decisions made by the routing protocol. Hence, to stop using a face, a static forwarding strategy requires the routing protocol to remove the face from the FIB or to find a lower cost face.

We describe two use cases to demonstrate the impact of different design approaches and the selected strategy on the application's correctness and performance.

\section{Use Case 1 - Multiple Producers.}

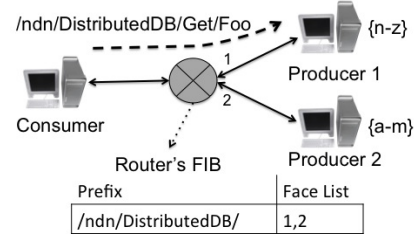

Figure 1: Multiple Producers with Disjoint Content

Figure 1 illustrates a distributed application consisting of multiple producers with disjoint content. Here the namespace is crucial and the strategy selection can play a critical role. When both producers publish the same namespace ndn/DistributedDB, the namespace entry in the router's FIB consists of both faces. If the namespace ndn/DistributedDB is paired with a static strategy, such as best-route [2], and 
face 1 is selected as the best face, then requests for Foo will be forwarded to producer 1 even though it cannot satisfy them. A static strategy will change its preferences in case of a link failure, but in our case, the link to 1 stays alive, and therefore face 2 will never get selected as the new best face. To solve this, the developer should design the application to use producer-specific namespaces instead of a general one. Alternatively, the developer must pair the application namespace with a strategy that guarantees a different behavior. One choice could be the broadcast [2] strategy. However, such a strategy will flood the network, and therefore is not advised. In this case, an adaptive strategy such as the default CCNx [2]strategy, or the Green-Yellow-Red [4] strategy will be the preferable choice.

\section{Use Case 2 - Notification Services.}

Notification service applications are distributed applications, in which the application's parties do not have clear consumer-producer relations and the distributed parties make requests to inform one another regarding their status.

We describe an example of a synchronization application that sends requests to synchronize the contents of a directory. Whenever a change is made to the synced directory, a participant sends a notification in the form of an Interest packet to inform the others of the change. Here, there are two possible approaches to designing the Interest namespace. The first approach is to make the participant identification part of the routable prefix. In this case, each participant has to learn about the existence of the others, so that he or she can send a dedicated interest packet, consisting of the participant identification, to each party.

In the second approach, the application uses the same namespace to send notice of a change to all the participants. Here, the selection of the forwarding strategy has a great impact on the application performance. A forwarding strategy that chooses a single face, such as best-route, forwards the Interest to only one participant, and therefore other participants are out-of-date. Alternatively, the broadcast strategy forwards the Interest to all the available faces of the namespace, and thereby guarantees that the notification message is forwarded to all the participants.

The CCNx Sync protocol [3] suggests an interesting design approach to a similar problem. The protocol uses the default $C C N x$ strategy to notify all the participants by sending a single Interest packet. The strategy selects one face when forwarding an Interest, but retransmits the Interest on additional faces if the Interest timer expired before the Data packet was received. In the CCNx Sync protocol, the participants do not reply to the notification Interest with a Data packet, and therefore the Interest always expires and is retransmitted to the next available face by the strategy.

\section{EMPIRICAL RESULTS}

We used four hosts in the Open Network Lab (ONL) [5] and NFD version 0.3 to experiment with the topology described in Figure 1. We set the consumer to request 100 chunks of content, with approximately $50 \%$ of the requests for content held by producer 1 , and the rest held by producer 2. We repeated the experiment using three strategies implemented in NFD 0.3 (ncc, best-route and broadcast), and reported the average number of Interests sent as shown in Figure 2a.

The broadcast strategy sent each Interest on both faces,

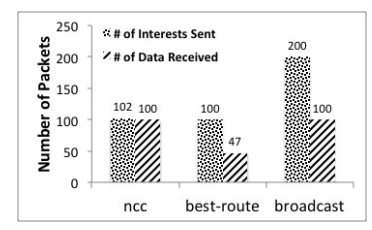

(a) Number of Interests Sent

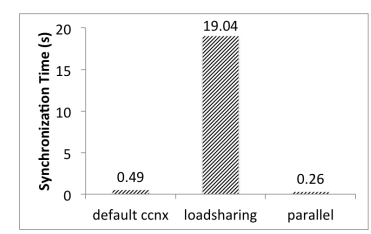

(b) Average Synchronization Time
Figure 2: Use Cases Empirical Results

and therefore sent twice the number of requests. The results show that best-route retrieved only $47 \%$ of the content items while broadcast and ncc successfully retrieved all the content items. Here, the results demonstrate how the application's correctness is affected by the forwarding strategy.

To demonstrate the second use case, we used 16 ONL hosts, each running $\mathrm{CCNx}$ version 0.8 .2 , to measure the performance of CCNx Sync when using different forwarding strategies as shown in Figure $2 b$. We inserted a content item into the repository of one of the 16 hosts and measured the average synchronization time using different forwarding strategies over a fully connected mesh topology. As expected, the parallel strategy achieved the shortest synchronization times, since the notification was multicasted to all the distributed parties simultaneously. The default $C C N x$ strategy was 1.8 times slower, and the loadsharing strategy was 73.1 times slower. Clearly the selected forwarding strategy significantly impacts the application's performance.

\section{CONCLUSION AND FUTURE WORK}

In this paper, we discussed the impact of the application triangle (the namespace design, the forwarding strategy and the application workflow) on the application's correctness and performance. We demonstrated that while different applications have different needs, and therefore use different design approaches, the application developer must consider the outcomes of selecting one way over another.

In future work, we would like to investigate real-world applications built on top of NDN. While a developer can select the strategies used in a closed environment, the network operator determines the forwarding strategies in the core network. Therefore, we would like to explore the relationship of forwarding strategies in different locations of the network. Another interesting question is whether one strategy outperforms others for a wide range of applications, and so can be used as the default forwarding strategy for NDN.

\section{REFERENCES}

[1] Lixia Zhang et al. Named data networking. ACM SIGCOMM Computer Communication Review, 2014.

[2] Alexander Afanasyev et al. Nfd developer's guide. Technical report, NDN-0021, NDN, 2014.

[3] Project CCNx. http://www.ccnx.org/.

[4] Cheng Yi et al. A case for stateful forwarding plane. Computer Communications, 2013.

[5] Charlie Wiseman et al. A remotely accessible network processor-based router for network experimentation. In Proceedings of the 4 th ACM/IEEE Symposium on Architectures for Networking and Communications Systems. ACM, 2008. 